\title{
Bio-Inspired Tactile Sensor Sleeve for Surgical Soft Manipulators
}

\author{
S. Sareh, A. Jiang, A. Faragasso, Y. Noh, T. Nanayakkara, Member, IEEE, \\ P. Dasgupta, L.D. Seneviratne, Member, IEEE, H.A. Wurdemann, K. Althoefer, Member, IEEE
}

\begin{abstract}
Robotic manipulators for Robot-assisted Minimally Invasive Surgery (RMIS) pass through small incisions into the patient's body and interact with soft internal organs. The performance of traditional robotic manipulators such as the da Vinci Robotic System is limited due to insufficient flexibility of the manipulator and lack of haptic feedback. Modern surgical manipulators have taken inspiration from biology e.g. snakes or the octopus. In order for such soft and flexible arms to reconfigure itself and to control its pose with respect to organs as well as to provide haptic feedback to the surgeon, tactile sensors can be integrated with the robot's flexible structure. The work presented here takes inspiration from another area of biology: cucumber tendrils have shown to be ideal tactile sensors for the plant that they are associated with providing useful environmental information during the plant's growth. Incorporating the sensing principles of cucumber tendrils, we have created miniature sensing elements that can be distributed across the surface of soft manipulators to form a sensor network capable of acquire tactile information. Each sensing element is a retractable hemispherical tactile measuring applied pressure. The actual sensing principle chosen for each tactile makes use of optic fibres that transfer light signals modulated by the applied pressure from the sensing element to the proximal end of the robot arm. In this paper, we describe the design and structure of the sensor system, the results of an analysis using Finite Element Modeling in ABAQUS as well as sensor calibration and experimental results. Due to the simple structure of the proposed tactile sensor element, it is miniaturisable and suitable for MIS. An important contribution of this work is that the developed sensor system can be "loosely" integrated with a soft arm effectively operating independently of the arm and without affecting the arm's motion during bending or elongation.
\end{abstract}

\section{INTRODUCTION}

In Robot-assisted Minimally Invasive Surgery (RMIS), robotic tools enter the body through narrow openings and manipulate soft organs that can move, deform, or change in stiffness. Surgical systems such as the da Vinci system that make use of the traditional robotic manipulation concept relying on rigid links without integrated force or tactile sensors have limitations on these laparoscopic and robotassisted surgical procedures due to restricted access through

S. Sareh, A. Jiang, A. Faragasso, Y. Noh, T. Nanayakkara, L.D. Seneviratne, H.A. Wurdemann, and K. Althoefer are with the Centre for Robotics Research, Department of Informatics, School of Natural and Mathematical Sciences, King's College London, Strand, London WC2R 2LS, UK. sina.sareh, allen.jiang, angela.faragasso, yohan.noh,

thrish.nanayakkara, lakmal.seneviratne,

helge.wurdemann, kaspar.althoefer@kcl.ac.uk

L.D. Seneviratne is with the Khalifa University Robotics Institute, Khalifa University, Abu Dhabi, United Arab Emirates. lakmal.seneviratne@kustar.ac.ae

P. Dasgupta is with the Department of Urology, Guy's and St Thomas' Hospitals, NHS Foundation Trust, Guys Hospital, London SE1 9RT, UK. prokarurol@gmail.com

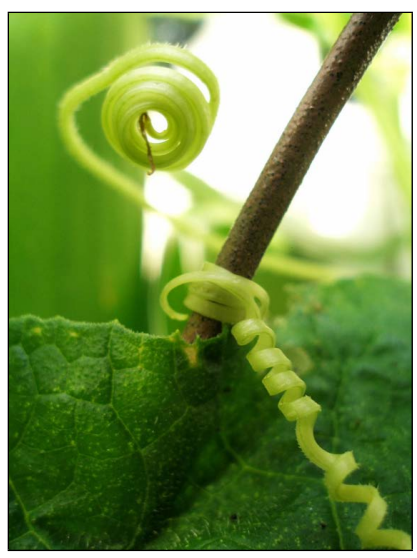

(a)

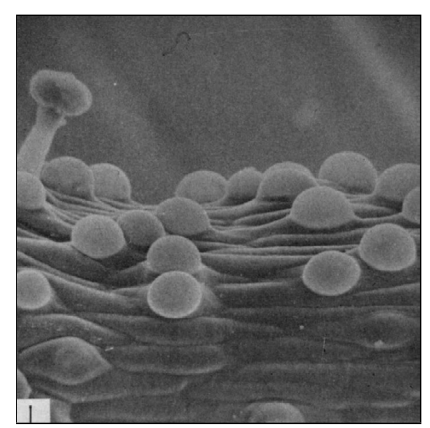

(b)
Fig. 1. (a) Cucumber tendril and (b) micrograph of the ventral side of tendrils [6] which shows Trichoma and Papillae-like tactile sensors

Trocar ports, lack of haptic feedback [1], [2], and difficulties with rigid robot tools operating inside a confined space filled with organs [3]. The solid structure of the instrument attached to the robotic arms and their external articulation make it problematic to change surgical targets inside the abdomen. In the case of the da Vinci Surgical System, the surgeons are not provided with haptic feedback from the interaction between the powerful and rigid robot arms and the patient's tissue - tissue tears can occur as a result [3], [4].

Nature has provided a rich source of inspiration for robotic scientist wishing to design flexible manipulators [5]. In particular, the patterns and mechanisms of movement are significant features of microorganisms, animals, and plants and, hence, a major focus of bio-inspired and bio-mimetic engineering [7]. These biological mechanisms are capable of manipulation inside unstructured and confined spaces and can act as ideal sources of inspiration for the design of surgical devices with enhanced maneuverability, safety and intractability. Featuring a high degree of controlled flexibility in their bodies, these biological systems are highly adaptable and interact safely with each other and with the environment [8]. So far, inspiration for surgical tools has been taken from snakes [9] and octopuses [10], [11]. In order for such soft and flexible arm to reconfigure itself and to control its pose with respect to organs as well as to provide haptic feedback to the surgeon, tactile sensors can be integrated with the robot's flexible structure. Depending on the characteristics of the surgical robot arm, tactile sensors need to accommodate certain features in order to complement 
the manipulator's structure.

The work presented here takes inspiration from another area of biology: cucumber tendrils have shown to be ideal tactile sensors for the plant that they are associated with providing useful environmental information during the plant's growth. The presented sleeve will be developed by considering the features of the soft modular manipulator which is inspired by the octopus morphology for RMIS in [11]. The manipulator is a novel cylinder-shaped device which is manufactured from Ecoflex Supersoft silicone with a Shore hardness of 0050A (Ecoflex 00-50) and covered by an off-the-shelf flexible braided sheath. This structural design allows omnidirectional bending, elongation capability, selective stiffness changing, and passive squeezing when pneumatically actuated via integrated chambers within the cylinder. The device has an overall length of $50 \mathrm{~mm}$ and an outer diameter of $35 \mathrm{~mm}$ with a minimum elongation capability of $30 \%$. Section II will review tactile sensors for stretchable and bendable devices. In Section III, the key objectives of the proposed tactile sensor properties will be summarised. Inspiration will be taken from the sensing mechanism in cucumber tendrils. The design of the bio-inspired tactile sensor sleeve will be presented (Section IV). A numerical analysis will be described in Section V. The calibration process in Section VI and the experimental results show the potential of this design concept.

\section{BACKGROUND}

Researchers have thoroughly explored tactile sensing systems capable of sensing only at robot's end-effector. These include haptic approaches for environmental exploration [12], [13], objects exploration [14], [15], feature detection e.g. bumps and ridges [16], object recognition [17] and estimation of object properties [18]. A comprehensive review can be found in [19].

Distributed tactile sensing along whole robotic manipulators facilitates distinguishing between distinct force distribution and contact configuration. For instance, a force in a surgical robot distributed over a small area produces higher stress and, therefore, higher potential of damaging organs than the same force distributed over a large area. Also, distributed tactile sensors can provide useful information on the geometry and texture of the contact [20], [21].

A tactile sensing sleeve incorporated to the body of a soft manipulator is required to be stretchable and conformable. In [22], a flexible and stretchable tactile skin composed of conductive fabric and urethane foam is demonstrated. However, this work does not discuss the sensor output when the tactile skin is stretched. Some tactile sensors reported in the literature are flexible, but not stretchable including the distributed tactile sensor made of conductive fabric and gel [23]. Examples of stretchable tactile sensors include a stretchable tactile sensor based on static electricity [24], a deformation sensitive tactile distribution sensor exploiting EIT (Electrical Impedance Tomography), which can estimate the resistivity distribution within an electrical conductor through measurements from its boundary [25], a stretchable and multifunctional capacitive sensor composed of gold thin films embedded in rubber [26], and a stretchable skinlike tactile sensor based on polymeric composites [27], and sensors based on liquid embedded microchannels [28]. The EIT based sensing systems consist of electrodes located on boundaries of the sensing mechanism. Hence, these systems cannot provide sufficient information about force distribution, unless a method of "multiple internal electrodes" is used [29]. Although the flexibility of the sensing mechanism based on gold-trace stripes and silicone materials in [26] allows bending and stretching of the sensing device, poor adhesion between the silicone substrate and thin gold films together with low electrical conductivity of film materials limit the integration of sensor arrays. The sensing system in [29] is composed of a transparent elastomer film embedded in micro-channel of conductive liquid (eGaIn). The sensing elements exert pressure on the embedded microchannel when bending the sensor. The change in the crosssection area of the micro-channel leads to a change in electrical resistance. Wang et al. [20] developed an array of dome-shaped patterned IPMCs based on Flemion, the perfluorocarboxylic acid membrane made by Asahi Glass Company, taking bio-inspiration from the tactile sensor cells on the skin of a cucumber tendril. Although, this sensing mechanism is particularly attractive due to its capability in sensing multi-degree-of-freedom forces, this sensing device is not stretchable.

\section{OBJECTIVES AND BIO-INSPIRATION}

To ensure unique contribution to science and to develop a novel sensor design and structure which complements the characteristics of the manipulator in [11], the following functional requirements are considered for the proposed tactile sensor sleeve:

1) A single tactile sensor of the sleeve should not be affected by features of the robot arm such as stiffening, bending and elongation.

2) For RMIS, the tactile sensor array will need to be designed for a manipulator diameter of up to $15 \mathrm{~mm}$. A simple and uncomplicated design of the sensor will allow the possibility of miniaturisation. A low-cost disposable steering mechanism would be another nonobligatory but important objective in RMIS.

In this paper, these requirements have been taken into account. The scientific contribution of the design of this tactile sensor array for soft manipulators will be evaluated by considering the achievements of these objectives.

As mentioned in Section I, nature has inspired the development of robotic devices in many ways. Here, we take inspiration of cucumber tendrils. Fig. 1(a) shows a cucumber tendril hooking around its host. Tendrils are used by climbing plants for support and attachment, generally by twining around suitable hosts. Rough surfaces are easier to grab on and, hence, better host candidates. The tactile papillae on tendrils which are shown in Fig. 1(b) are only 0.8-2.0 $\mu \mathrm{m}$ thick. The biological sensors have a simple dome-shaped 
structure. The thickness of the wall is only between 0.3$0.8 \mu \mathrm{m}$. Researchers conclude that these papillae function as tactile receptors [6]. The structure and functionality as a biological receptor benefits and, thus, is considered for the development of the tactile sensor sleeve for RMIS.

\section{DESIGN OF THE BIO-INSPIRED TACTILE SENSOR ARRAY}

Inspired by the structure of the distributed tactile sensing units on the surface of cucurbitaceae, this section explains the design and fabrication of a sleeve with integrated tendrilinspired tactile sensors. The individual dome-shaped sensors are produced using two different materials. Their performance will be analysed and compared in Section V and VI. The tactile sensors are based on fibre-optic light modulation. Multiple sensors are then integrated into a silicone sleeve.

\section{A. Bio-Inspired Tactile Sensor Design}

The design of the proposed individual tactile sensors is closely influenced by the tactile papillae in Fig. 1(b). Fig. 2(a) illustrates the assembly of the bio-inspired sensor. The base has a $10 \mathrm{~mm}$ diameter and $1 \mathrm{~mm}$ thickness with a convex surface to be aligned along the manipulator. It is manufactured using a rapid prototyping machine (Project HD-3000 Plus, 3D Systems). The Project rapid prototyping machine employs a large number of printing jets to print objects in 3D. Using this machine to manufacture the novel segment structure of the manipulator allows miniaturisation. The polymer material used to print the base is nonconductive ABS polymer (Acrylonitrile Butadiene Styrene). A flexible hemi-spherical dome of $8 \mathrm{~mm}$ diameter and $4 \mathrm{~mm}$ height (thickness: $1 \mathrm{~mm}$ ) is mounted on the base. In this paper, we fabricated two sets of domes using two different materials: Poly PT Flex rubber with a Shore hardness of 70A and Ecoflex Supersoft silicone with a Shore hardness of 00-50A. The properties of the two materials are listed in Table I.

The proposed tactile sensor uses light intensity modulated fibre-optics [30]-[32] based on a prismatic tip. Hence, the base is designed so that it embeds a $1 \mathrm{~mm}$ fibre as shown in Fig 2(a). A mirror is fitted inside the dome which reflects emitted light by the fibre. The intensity of the reflected light will vary when the distance between the fibre tip and mirror changes due to forces applied to the dome. The fibre tip is cut at $45^{\circ}$ angle and polished in order to provide an internal

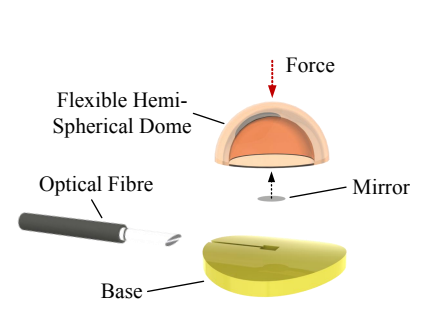

(a)

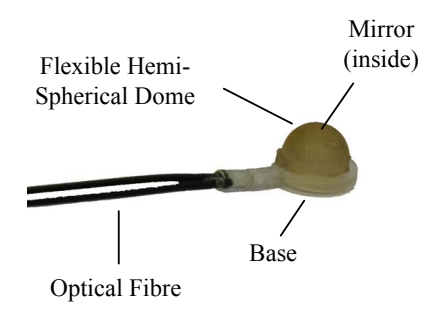

(b)
Fig. 2. (a) CAD drawing and (b) prototype of an assembled single tactile sensor

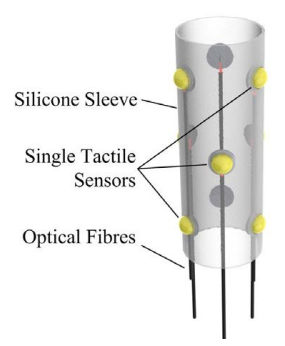

(a)

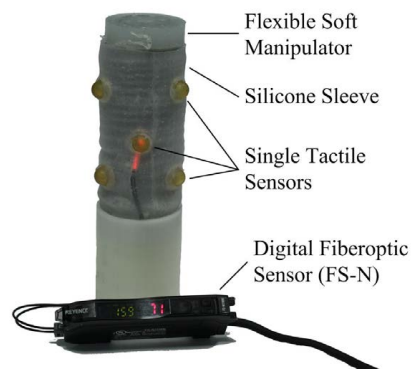

(b)
Fig. 3. (a) CAD drawing and (b) prototype of the sensor sleeve with multiple tactile sensor

reflection of $90^{\circ}$. The light is moving inside the fibre until it reaches the prismatic tip and changes its direction by $90^{\circ}$ before leaving the fibre. Inversely, when the light is reflected back, the light changes the direction by $90^{\circ}$ once it hits the prismatic tip and travels along the length of the fibre. The utilisation of the prismatic tip fibre ensures a planar structure of the sensor design. More details about this sensing mechanism can be found in [33]. A prototype with a hemispherical dome made of polyurethane rubber is shown in Fig. 2(b).

\begin{tabular}{l|c|c} 
Material & $\begin{array}{c}\text { Elongation } \\
\text { at Break }\end{array}$ & $\begin{array}{c}\text { Shore A } \\
\text { Hardness }\end{array}$ \\
\hline \hline $\begin{array}{l}\text { Ecoflex Supersoft } \\
\text { Silicone }\end{array}$ & $980 \%$ & $00-50$ \\
\hline Poly PT Flex Rubber & $175 \%$ & 70
\end{tabular}

TABLE I

MATERIAL PROPERTIES FOR THE TWO SETS OF THE SENSOR DOME

\section{B. Integration of Multiple Sensors}

As mentioned earlier, the entire robot arm is fabricated from Ecoflex 00-50. A braided polyester sheath is used for the outer membrane which only allows longitudinal extension of the manipulator.

A sleeve has been designed as shown in Fig. 3(a) to embed tactile sensors. Ecoflex 00-50 is moulded in a cylindrical shaped tube with a thickness of $1 \mathrm{~mm}$. In order to fit the stretchable sleeve to the manipulator, the silicone mould has a $30 \mathrm{~mm}$ diameter and $45 \mathrm{~mm}$ length. In this paper, 9 sensors are fixed into equally distributed holes of $7 \mathrm{~mm}$ diameter along the sleeve. Fig. 3(b) shows an assembled prototype with rubber-dome sensors embedded into the sensor sleeve. The optical fibres are connected to a Keyence Digital Fibreoptic Sensor which measures the light intensity variation between the emitted and received light when applying a force to the sensor. In order to feed the optical fibres during bending and elongation, the fibres are radially aligned into the gaps of the crimped sheath.

\section{FINITE ELEMENT ANALYSIS}

A Finite Element Model (FEM) of the entire structural system has been set up in order to compare the behaviour 
of the two types of individual bio-inspired sensors (Poly PT Flex rubber and Ecoflex Supersoft silicone) when subjected to displacement and traction force. The analysis was run using the commercial FE software ABAQUS/Explicit 6.123. The parts were created using SolidWorks Simulia and imported to ABAQUS for mesh generation, material property assignment and stress analysis. The focus was then shifted to a unit cell which faithfully represents the whole model in all its important features. As the focus is on the comparative study between two cases, the curved shell element base of the single sensor is replaced by a continuum plate part of Ecoflex Supersoft silicone. The FEM of the single tactile sensor has been studied and the evolution of stress and strain in the part is depicted. Fig. 4(a) and 4(b) show the contour of von Mises stress of a in-plane loaded tactile sensor unit. It can be conspicuously seen that when the homogeneous part (in case of using Ecoflex Supersoft silicone for the sensor dome) is loaded in plane the hemispherical outgrowth deforms substantially and takes the form of an ellipsoid with the largest axis of the base cross section aligning with the direction of tensile load. When on the other hand the heterogeneous model (in case of using Poly PT Flex rubber for the sensor dome) is loaded in plane, no considerable deformation of the hemisphere is observed. These results are well in line with intuition.

\section{CALIBRATION AND EXPERIMENTAL RESULTS}

\section{A. Experimental Setup}

To calibrate the light intensity of the tactile sensor with applied force, experiments were performed on the Ecoflex Supersoft silicone and Poly PT Flex rubber sensors. The experiment consisted of loading and unloading the tactile sensor with a $4 \mathrm{~mm}$ deflection (see Fig. 5). The resulting load force was measured by an ATI Nano17 Force/Torque

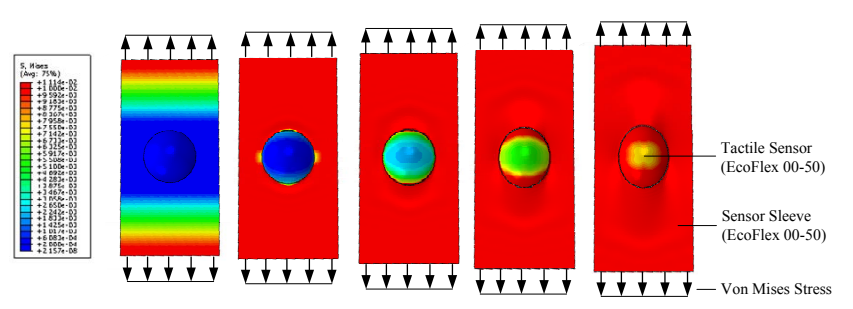

(a)

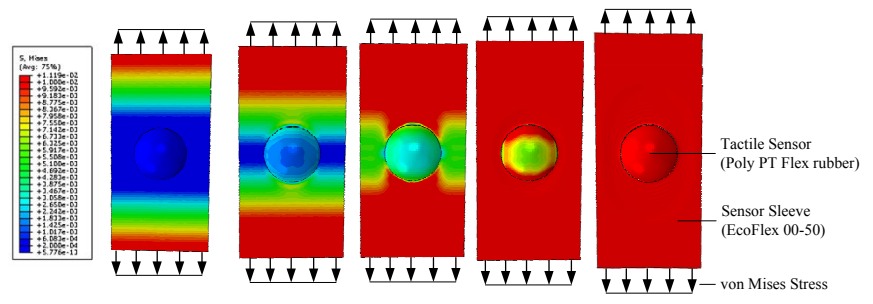

(b)

Fig. 4. Mises stress of in-plane loaded unit cells for an individual tactile sensor using (a) Ecoflex Supersoft silicone and (b) Poly PT Flex rubber implemented into the silicone sleeve sensor. The loading phase was controlled by a motorised linear module at a speed of $0.25 \frac{\mathrm{mm}}{\mathrm{s}}$. At the $4 \mathrm{~mm}$ deflection point, the module pauses for $5 \mathrm{~s}$ before transitioning to the unloading phase. The unloading phase is identical to the load phase, with the exception that the module is moving in the opposite direction to its initial position. Data from the force sensor and light intensity meter were recorded through a DAQ card at $1 \mathrm{kHz}$. Five trials were performed for each sensor type, and with the average and variability of each point plotted against the deflection distance. Hysteresis was calculated by taking the area between the loading and unloading curves, and normalizing it by dividing it by the loading curve.

\section{B. Calibration Results}

Fig. 6(a) shows the load and unload forces measured by the force sensor, and Fig. 6(b) shows the light intensity measured by the light intensity meter. For both the rubber and silicone tactile sensors, saturation occurs from the $2.8 \mathrm{~mm}$ point, and thus higher forces were not recorded. From Fig. 7(a), the tactile sensor made of Poly PT Flex rubber exhibited a fairly linear profile, reaching a peak of $11.70 \mathrm{~N}$ with a hysteresis value of 0.50 . The sensor made of Ecoflex Supersoft silicone displayed a nonlinear behaviour, and had a peak force of $4.50 \mathrm{~N}$ with a hysteresis value of 0.44 . The average variability of the rubber and silicone sensors were 0.83 and $0.36 \mathrm{~N}$, respectively.

The light intensity data in Fig. 7(b) are similar to Fig. 7(a), though exhibit more nonlinear behaviour. Fig. 8(a) shows the Ecoflex Supersoft silicone calibration curve between the measured light intensity and force as the sensor is pressed. The profile is linear between 1080 and 1260 Lux (0.38 to $0.57 \mathrm{~N}$ ). For the Poly PT Flex rubber calibration curve, as seen in Fig. 8(b), the linear region occurs between 2300 and 3740 Lux ( 3.71 to $7.24 \mathrm{~N}$ ). Thus, the stiffness of the tactile sensor material affects the measurable force range; the stiffer polyurethane rubber is not only capable of measuring higher forces, but with a wider range, as well. It should be noted

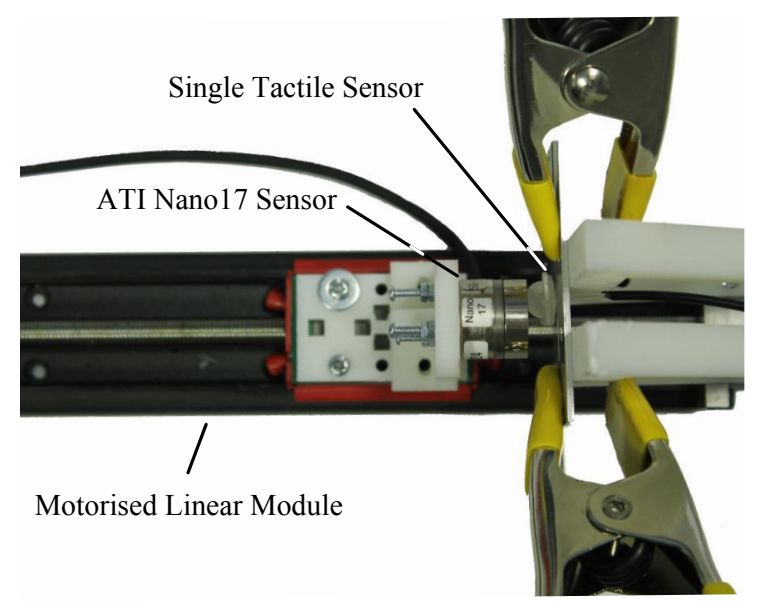

Fig. 5. Experimental setup for sensor calibration with a ATI Nano17 Force/Torque sensor using a motorised linear module 


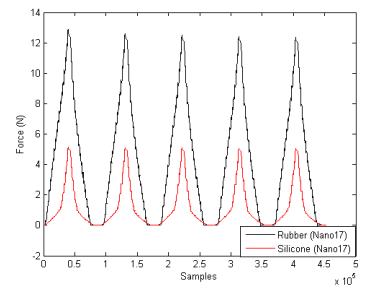

(a)

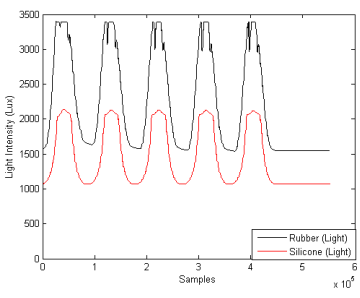

(b)
Fig. 6. Raw data for (a) force and (b) light intensity for a single tactile sensor

that the light intensity for the silicone and rubber sensors do not exhibit at the same value at zero force. This is due to the light reflectivity of the materials; the rubber reflects more ambient light than the silicone.

\section{Experimental Elongation and Bending Behaviour}

The sensing sleeve with integrated bio-inspired receptors using Poly PT Flex rubber hemi-spherical domes is fitted to the entirely soft manipulator presented in [11]. Fig. 9 shows the results during elongation and bending. In the relaxed configuration, the manipulator has a length of ca. $50 \mathrm{~mm}$. The manipulator is pneumatically actuated to a length of ca. $64 \mathrm{~mm}$ by $28 \%$. The sleeve stretches consistently and re-positions each single tactile sensor without affecting its shape. The right image of Fig. 9 shows how the tactile sensor sleeve allows the manipulator to bend.

In order to compare an integrated Ecoflex Supersoft silicone and Poly PT Flex rubber dome when subject to stress, the sleeve with three tactile sensors is loaded as shown in Fig. 10. The sleeve is elongated from its relaxed state of $60 \mathrm{~mm}$ to about $80 \mathrm{~mm}$ by over $30 \%$. The experimental results confirm the Finite Element Analysis in Section V that the heterogeneous sensor sleeve show no considerable deformation of the hemi-spherical domes.

\section{CONCLUSIONS AND FUTURE WORK}

This paper has presented a flexible and stretchable tactile sensor sleeve for modern soft manipulators in RMIS. The structure and functionality of tactile papillae on cucumber tendrils inspired the design of a single tactile sensor. Two sets of sensors were assembled using two different materials for the sensing dome: Poly PT Flex rubber with a Shore hardness of 70A and Ecoflex Supersoft silicone with a Shore hardness

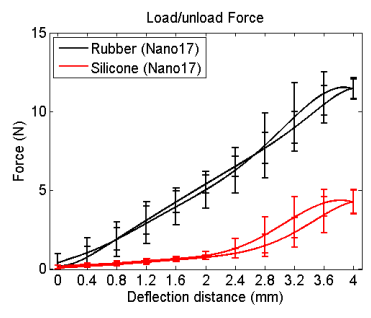

(a)

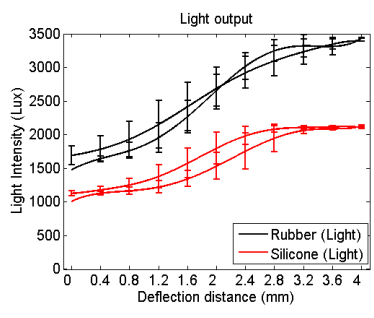

(b)
Fig. 7. Deflection versus (a) force and (b) light intensity for a single tactile sensor

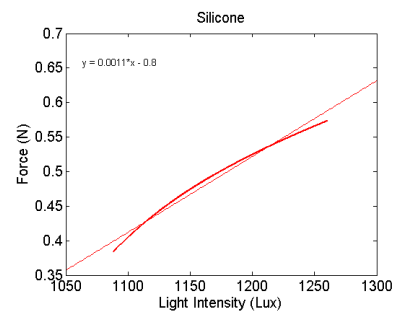

(a)

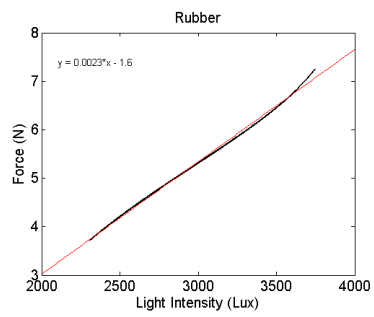

(b)
Fig. 8. Calibration curves: Light intensity versus force for (a) a Ecoflex Supersoft silicone and (b) Poly PT Flex rubber hemi-spherical dome

of 00-50A. The integration of multiple individual receptors into a silicone sleeve made of Ecoflex Supersoft silicone has been described. The sensing technique is based on light intensity modulation using fibre optics with a prismatic tip. The Finite Element Analysis shows no considerable deformation of the hemi-spherical dome in case of using Poly PT Flex rubber when applying stress to the sensing sleeve. This was confirmed during experimental elongation and bending tests as well. The calibration of the sensor results in a linear behaviour within a force range for the two different kind of tested materials. However, the sensing dome of Poly PT Flex rubber shows a higher force range and less hysteresis. In the current design, materials have only be used that have no effect on the homogeneity of MR images which makes this device MR-compatible.

Future work will expand the potential of this new stretchable sensor and investigate different types of silicone to identify wider force ranges and allow even more elongation of the manipulator. Another focus will be the influence of the tactile sensor sleeve on changes to the manipulator's kinematics. Further miniaturisation is needed in order to use the sensing sleeve for RMIS.

\section{ACKNOWLEDGEMENT}

The work described in this paper is supported by STIFFFLOP project grant from the European Communities Seventh Framework Programme under grant agreement 287728. The research was also partially funded/supported by the National Institute for Health Research (NIHR) Biomedical Research Centre based at Guy's and St Thomas' NHS Foundation Trust and King's College London. The views expressed are those of the authors and not necessarily those of the NHS, the NIHR or the Department of Health.
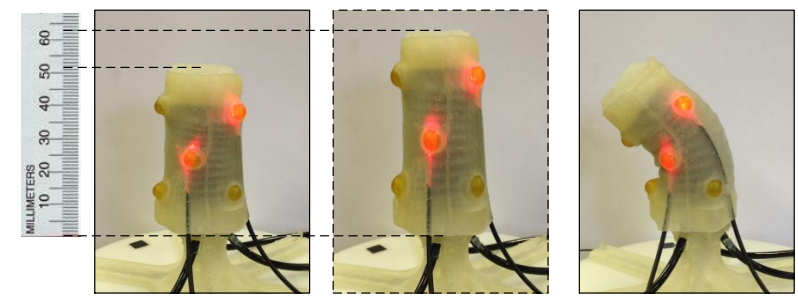

Fig. 9. Tactile sensor sleeve behaviour during manipulator elongation and bending 


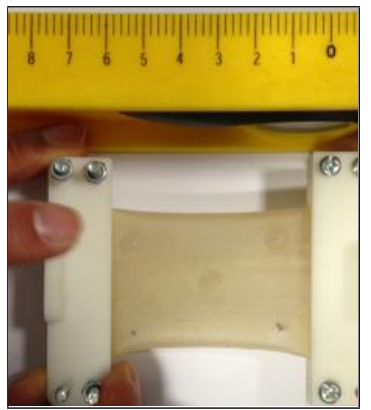

(a)

(c)

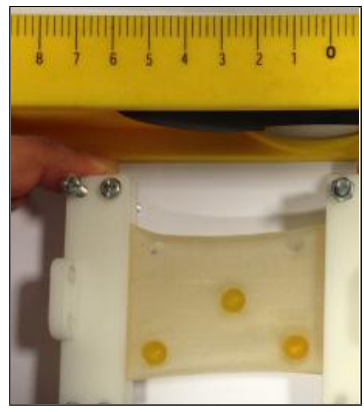

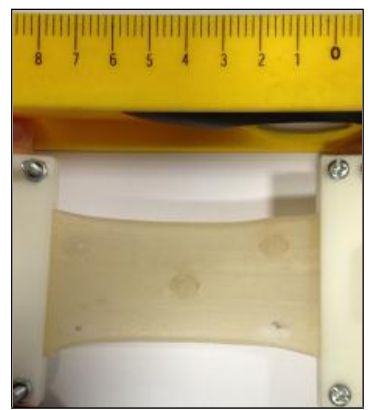

(b)

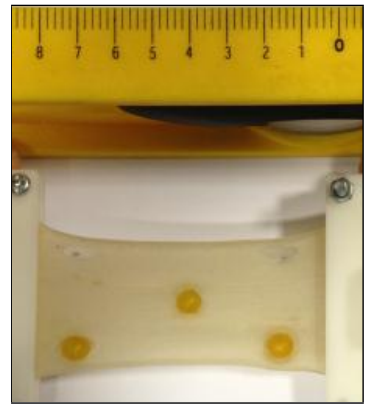

(d)
Fig. 10. Stress analysis for tactile sensors with (a) (b) Ecoflex Supersoft silicone and (c) (b) Poly PT Flex rubber hemi-spherical dome

\section{REFERENCES}

[1] G. Dogangil, B. Davies, and F. R. y Baena, "A review of medical robotics for minimally invasive soft tissue surgery," Proceedings of the Institution of Mechanical Engineers Part H: Journal of Engineering in Medicine, vol. 224, pp. 653-679, 2010.

[2] K. Sangpradit, H. Liu, L. Seneviratne, and K. Althoefer, "Tissue identification using inverse finite element analysis of rolling indentation," in IEEE International Conference on Robotics and Automation, pp. 1250-1255, 2009.

[3] H. Wurdemann, A. Jiang, T. Nanayakkara, L. Seneviratne, and K.Althoefer, "Variable stiffness controllable and learnable manipulator for mis," in IEEE International Conference on Robotics and Automation Workshop: Modular Surgical Robotics: how can we make it possible?, 2012.

[4] M. Li, H. Liu, J. Li, L. D. Seneviratne, and K. Althoefer, "Tissue stiffness simulation and abnormality localization using pseudo-haptic feedback," in IEEE International Conference on Robotics and Automation, pp. 5359-5364, 2012.

[5] S. Sareh, J. Rossiter, A. Conn, K. Drescher, and R. Goldstein, "Swimming like algae: biomimetic soft artificial cilia," Journal of the Royal Society Interface, vol. 10, no. 78, 2012.

[6] S. Junker, "Ultrastructure of tactile papillae on tendrils of eccremocarpus scaber r. et p.," New Phytol., vol. 78, pp. 607-610, 1977.

[7] D. Trivedi, C. Rahn, W. Kierb, and I. Walker, "Soft robotics: Biological inspiration, state of the art, and future research," Applied Bionics and Biomechanics, vol. 5, no. 3, p. 99117, 2008.

[8] S. Sanan, M. Ornstein, and C. Atkeson, "Physical human interaction for an inflatable manipulator," in Engineering in Medicine and Biology Society, 2011.

[9] J. Shang, D. Noonan, C. Payne, J. Clark, M. Sodergren, A. Darzi, and G.-Z. Yang, "An articulated universal joint based flexible access robot for minimally invasive surgery," in IEEE International Conference on Robotics and Automation, pp. 1147-1152, 2011.

[10] C.Laschi, M. Cianchetti, B. Mazzolai, L. Margheri, M. Follador, and P. Dario, "Soft robot arm inspired by the octopus," Advanced Robotics, vol. 26, no. 7, pp. 709-727, 2012.

[11] M. Cianchetti, T. Ranzani, Member, G. Gerboni, I. de Falco, C. Laschi, and A. Menciassi, "STIFF-FLOP surgical manipulator: mechanical design and experimental characterization of the single module," in IEEE Conference on Intelligent Robots and Systems, 2013.
[12] G. Ganesh, N. Jarrasse, S. Haddadin, A. Albu-Schaefer, and E. Burdet "A versatile biomimetic controller for contact tooling and haptic exploration," in IEEE International Conference on Robotics and Automation, pp. 3329-3334, 2012.

[13] S. Haddadin, A. Albu-Schaefer, A. de Luca, and G. Hirzinger, "Collision detection and reaction: A contribution to safe physical humanrobot interaction," in IEEE/RSJ International Conference on Intelligent Robots and Systems, pp. 3356-3363, 2011.

[14] D. Kraft, A. Bierbaum, M. Kjaergaard, J. Ratkevicius, A. KjaerNielsen, and C. Ryberg, "Tactile object exploration using cursor navigation sensors," in EuroHaptics Symposium on haptic interfaces for virtual environment and teleoperator systems, p. 296301, 2009.

[15] A. Okamura and M. Cutkosky, "Feature detection for haptic exploration with robotic fingers," The International Journal of Robotics Research, vol. 20, pp. 925-938, 2001.

[16] A. Okamura, Haptic exploration of unknown objects. PhD thesis, Department of Mechanical Engineering, Stanford University, 2000.

[17] Z. Pezzementi, E. Plaku, C. Reyda, and G. Hager, "Tactile-object recognition from appearance information," IEEE Transactions on Robotics, vol. 27, no. 3, p. 473487, 2011.

[18] S. Chitta, J. Sturm, M. Piccoli, and W. Burgard, "Tactile sensing for mobile manipulation," IEEE Transactions on Robotics, vol. 27, no. 99, p. 111, 2011.

[19] P. Puangmali, K. Althoefer, L. Seneviratne, D. Murphy, and P. Dasgupta, "State-of-the-art in force and tactile sensing for minimally invasive surgery," IEEE Sensors Journal, vol. 8, no. 4, 2008.

[20] J. Wang, H. Sato, C. Xu, and M. Taya, "Bioinspired design of tactile sensors based on flemion," J. Appl. Phys., vol. 105, 2009.

[21] A. Jain, M. Killpack, A. Edsinger, and C. Kemp, "Reaching in clutter with whole-arm tactile sensing," The International Journal of Robotics Research, 2013

[22] T. Hoshi and H. Shinoda, "A sensitive skin based on touch-areaevaluating tactile elements," in Symposium on Haptic Interfaces for Virtual Environment and Teleoperator Systems, 2006.

[23] R. Tajima, S. Kagami, M. Inaba, and H. Inoue, "Development of soft and distributed tactile sensors and the application to a humanoid robot," Advanced Robotics, vol. 16, no. 4, p. 381397, 2002.

[24] Y. Tada, M. Inoue, T. Kawasaki, Y. Kawahito, H. Ishiguro, and K. Suganuma, "A flexible and stretchable tactile sensor utilizing static electricity," in IEEE/RSJ International Conference on Intelligent Robots and Systems, 2007.

[25] A. Nagakubo, H. Alirezaei, and Y. Kuniyoshi, "A deformable and deformation sensitive tactile distribution sensor," in IEEE International Conference on Robotics and Biomimetics, 2007.

[26] D. Cotton, I. Graz, and S. Lacour, "A multifunctional capacitive sensor for stretchable electronic skins," IEEE Sensors Journal, vol. 9, no. 12, 2009.

[27] L. Ventrelli, L. Beccai, V. Mattoli, A. Menciassi, and P. Dario, "Development of a stretchable skin-like tactile sensor based on polymeric composites," in IEEE International Conference on Robotics and Biomimetics, 2009.

[28] C. Majidi, R. Kramer, and R. Wood, "A non-differential elastomer curvature sensor for softer-than-skin electronics," Smart Mater. Struct., vol. 20, p. 105017, 2011 .

[29] D. Tawil, D. Rye, and M. Velonaki, "Improved image reconstruction for an eit-based sensitive skin with multiple internal electrodes," IEEE Transactions on Robotics, vol. 27, no. 3, pp. 425 - 435, 2011.

[30] P. Polygerinos, P. Puangmali, T. Schaeffter, R. Razavi, L. Seneviratne, and K. Althoefer, "Novel miniature mri-compatible fiber-optic force sensor for cardiac catheterization procedures," in IEEE International Conference on Robotics and Automation, pp. 2598-2603, 2010.

[31] P. Puangmali, H. Liu, L. Seneviratne, P. Dasgupta, and K. Althoefer, "Miniature 3-axis distal force sensor for minimally invasive surgical palpation," IEEE/ASME Transactions on Mechatronics, vol. 17, no. 4 pp. 646-656, 2012.

[32] H. Xie, A. Jiang, H. Wurdemann, H. Liu, L. Seneviratne, and K. Althoefer, "Magnetic resonance-compatible tactile force sensor using fiber optics and vision sensor,' IEEE Sensors Journal, vol. 14, no. 3 , pp. 829-838, 2013.

[33] A. Ataollahi, P. Polygerinos, P. Puangmali, L. Seneviratne, and K. Althoefer, "Tactile sensor array using prismatic-tip optical fibers for dexterous robotic hands," in IEEE/RSJ International Conference on Intelligent Robots and Systems, pp. 910,915, 2010. 